\title{
Light Transport and Localization in Two-Dimensional Correlated Disorder
}

\author{
Gaurasundar M. Conley, ${ }^{1,2}$ Matteo Burresi, ${ }^{1,3,}{ }^{*}$ Filippo Pratesi,${ }^{1}$ Kevin Vynck,,${ }^{1,4}$ and Diederik S. Wiersma ${ }^{1,3}$ \\ ${ }^{1}$ European Laboratory for Non-linear Spectroscopy (LENS), University of Florence, Via Nello Carrara 1, 50019 Sesto Fiorentino, Italy \\ ${ }^{2}$ Physics Department, University of Fribourg, Chemin du Musée 3, 1700 Fribourg, Switzerland \\ ${ }^{3}$ National Institute of Optics (CNR-INO), Largo Fermi 6, 50125 Florence, Italy \\ ${ }^{4}$ Institut Langevin, ESPCI ParisTech, CNRS, 1 rue Jussieu, 75238 Paris Cedex 05, France
}

(Received 23 July 2013; published 7 April 2014)

\begin{abstract}
Structural correlations in disordered media are known to affect significantly the propagation of waves. In this Letter, we theoretically investigate the transport and localization of light in 2D photonic structures with short-range correlated disorder. The problem is tackled semianalytically using the Baus-Colot model for the structure factor of correlated media and a modified independent scattering approximation. We find that short-range correlations make it possible to easily tune the transport mean free path by more than a factor of 2 and the related localization length over several orders of magnitude. This trend is confirmed by numerical finite-difference time-domain calculations. This study therefore shows that disorder engineering can offer fine control over light transport and localization in planar geometries, which may open new opportunities in both fundamental and applied photonics research.
\end{abstract}

DOI: 10.1103/PhysRevLett.112.143901

PACS numbers: 42.25.Dd, 42.25.Fx, 61.43.Dq

Multiple light scattering in disordered media plays a paramount role in the study of complex natural systems (e.g., biological tissues, porous materials, planetary atmospheres) [1] and wave phenomena (e.g., light localization, anomalous diffusion) [2-4]. In recent years there has been a growing interest in the use of photonic structures with controlled disorder, in particular within the context of mesoscopic transport effects [5-8], cavity quantum electrodynamics [9], photon management for energy efficiency [10-13], and even lab-on-chip spectroscopy [14]. Indeed structural correlations in the positions of scatterers are known to affect light propagation. Previous studies have shown that short-range correlations can either diminish or enhance the scattering strength of a disordered system [15-18] and lead to a modulation of the density of optical states [19], even in biological systems [20]. Such a modulation can be so large that a complete photonic band gap is expected to form, even without long-range periodicity [19,21-24]. The emerging concept of "disorder engineering" to manipulate light transport in random media is, however, still in its infancy and little is known so far on the occurrence of localization phenomena in correlated systems.

In this Letter, we theoretically investigate the transport of light and the occurrence of localization in two-dimensional (2D) photonic structures possessing short-range correlated disorder. A semianalytical model describing the wave propagation in correlated-disordered systems allows us to investigate how key quantities, namely, the transport mean free path, the scattering anisotropy factor, and the localization length, evolve with the degree of correlation. In particular, short-range correlations are found to allow for the tuning of the localization length over several orders of magnitude and thus, make it possible to go from a quasiextended to a strongly localized regime very easily, in sharp contrast with three-dimensional systems, where the localized regime is very difficult to reach $[8,25]$. This trend is confirmed by numerical simulations.

The 2D photonic structures consist of disordered patterns of circular air holes $\left(n_{i}=1\right)$ with filling fraction $f=20 \%$ and diameter $\varnothing=0.23 a$, where $a$ is the period of a hexagonal lattice of holes with the same $f$, in a medium with refractive index $n_{o}=3.5[10,19]$. The short-range correlation in the disorder is controlled by imposing a minimum distance $d_{\min }$ between the centers of the holes. This has been obtained $[10,26]$ by generating a disordered packing of hard disks with diameter $d_{\min }$ at a packing fraction $p$, using a freely available code [27] based on the Lubachevsky-Stillinger algorithm [28]. The centers of these disks have been used to generate the point patterns shown in Fig. 1(a) for three photonic structures with $p=30 \%, 50 \%, 70 \%$. The degree of local order evidently increases with increasing $p$, imposing an average distance between adjacent holes.

Fundamentally, the existence of a typical distance between neighboring scatterers implies a certain phase relation which, depending on direction and wavelength of scattered waves in the medium, gives rise to either constructive or destructive interference between them. Following this line of reasoning, structural correlations can be seen as a modification of the angular scattering pattern of the individual scatterers, and be taken into account (to a first approximation) by correcting the expression of the single scatterer differential scattering cross section $d \sigma / d \theta$ by the static structure factor $S(q)$ as $[15,16]$

$$
\frac{d \sigma^{\star}}{d \theta}=\frac{d \sigma}{d \theta} S(q),
$$



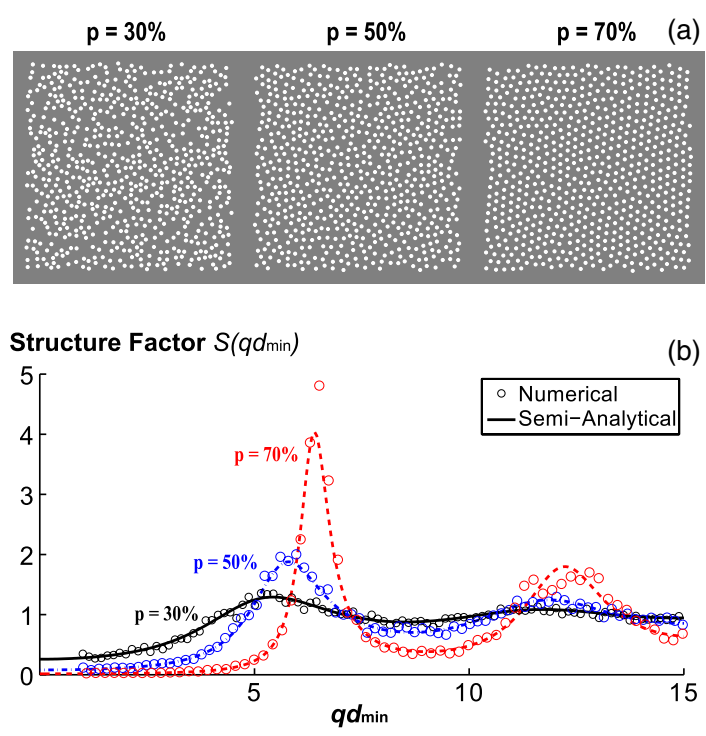

FIG. 1 (color online). Structural correlations in 2D disordered media. (a) Disordered photonic structures with filling fraction $f=20 \%$ and different degrees of correlation ( $p=30 \%, 50 \%$, $70 \%)$. (b) Structure factors $S\left(q d_{\min }\right)$ of the correlated patterns, evaluated numerically with Eq. (2) (hollow dots) and semianalytically using the Baus-Colot model (solid lines).

where $q=\left(4 \pi / \lambda_{e}\right) \sin (\theta / 2), \theta$ is the scattering angle, and $\lambda_{e}=\lambda / n_{e}$ is the wavelength in a medium with effective refractive index $n_{e}$, which in our case equals 2.92 according to the two-dimensional Maxwell Garnett mixing rule $[29,30]$. Formally, the structure factor $S(\mathbf{q})$ is defined as

$$
S(\mathbf{q})=\frac{1}{N}\left\langle\sum_{i, j=1}^{N} e^{-i \mathbf{q} \cdot\left(\mathbf{r}_{i}-\mathbf{r}_{j}\right)}\right\rangle,
$$

where $N$ is the number of scatterers, $\mathbf{r}_{i, j}$ the position of the scatterers labeled $i$ and $j$, and $\langle\ldots\rangle$ denotes ensemble average. In previous works on $2 \mathrm{D}$ photonic structures with short-range correlated disorder (statistically isotropic), $S(q)$ was calculated numerically from the point patterns generated by a sphere packing protocol [19]. This approach is very time consuming and not suited to an exhaustive study of the effect of structural correlations on transport. An analytical expression of the structure factor of a correlated-disordered medium is often retrieved by making use of the well-known PercusYevick model which, unfortunately, applies exclusively to systems with odd dimensionality $(d=1,3, \ldots)$ [31]. In contrast, we adopt a semianalytical approach, based on the Baus-Colot (BC) model for the structure factor of a fluid of hard disks [32], that is well-suited to systems of dimensionality $d=2$. In Fig. 1(b), we compare the structure factor $S\left(q d_{\min }\right)$ evaluated numerically using Eq. (2) for the $2 \mathrm{D}$ photonic structures generated above with those obtained from the $\mathrm{BC}$ model, using $p$ as the only input parameter. A very good agreement is observed, even for high $p$. As the degree of correlation is increased, the structure factor exhibits stronger oscillations, which indicate the emergence of a typical distance between neighboring scatterers.

By making use of Eq. (1), we calculate the angularly and spectrally resolved "effective" differential scattering cross section $d \sigma^{\star} / d \theta$ of holes in TE polarization (electric field in the plane) as a function of the degree of short-range correlation [Fig. 2(a)]. The single scatterer differential scattering cross section $d \sigma / d \theta$ was calculated from Mie theory for circular cylinders [33]. As $p$ increments, $d \sigma^{\star} / d \theta$ exhibits increasingly sharper features in frequency and angle due to the oscillations of $S(q)$, giving considerably different weights to the forward and backward scattering. Clearly, for low $p$ the scattering is primarily forward, whereas for strongly correlated disorder the forward scattering is inhibited in a broad range of frequencies.

This redistribution of the scattered light is at the core of the modification of the transport properties in correlated disorder. To illustrate this point, we calculate the transport mean free path $\ell_{t}$ in the correlated system [34]

$$
\ell_{t}=\left(\rho \int_{0}^{\pi} \frac{d \sigma^{\star}}{d \theta}(1-\cos \theta) d \theta\right)^{-1}
$$

where $\rho$ is the number density of scatterers, and the scattering anisotropy factor $g$

$$
g=\frac{1}{\sigma^{\star}} \int_{0}^{\pi} \frac{d \sigma^{\star}}{d \theta} \cos \theta d \theta
$$

which indicates the degree of anisotropy of the effective single scattering event. The results are shown in Figs. 2(b) and (c) as a function of $p$. First, as expected, correlations yield spectral ranges with either longer or shorter transport mean free paths, the latter occurring in particular when $\lambda_{e}=2 n d_{\mathrm{avg}}$, with $n=1,2, \ldots$ (Bragg-like scattering) and $d_{\text {avg }}$ is the average distance between nearest-neighbor scatterers. Variations larger than a factor of 2 are observed. Second, the anisotropy factor $g$ for highly correlated structures becomes negative on broad frequency ranges, reaching values as low as -0.9 , indicating a strong backward scattering [17]. Interestingly, this leads to a peculiar light transport in which the scattering mean free path $\ell_{s}=$ $\ell_{t}(1-g)$ is longer than the transport mean free path $\ell_{t}$. This scattering property is rare in systems of isolated particles, and it has been observed only in specific cases [35].

Gaining control over the transport mean free path provides an unprecedented control on light localization phenomena. In this respect, two-dimensional structures are very peculiar since the dependence of the localization length on the transport mean free path is critical, so that a small change in $\ell_{t}$ should yield dramatic changes of $\xi$. The localization length is indeed predicted to be given by [3]

$$
\xi \approx \ell_{t} \exp \left[\pi^{2} \frac{\ell_{t}}{\lambda_{e}}\right]
$$




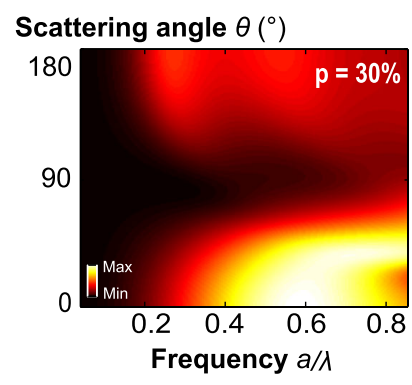

(a)

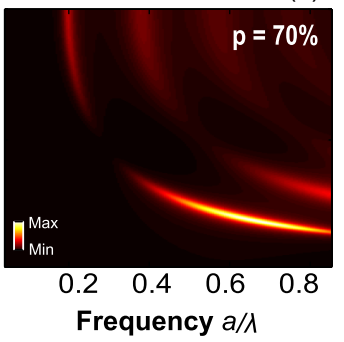

Inverse transport mfp $\ell_{t}^{-1}\left[a^{-1}\right]$

(b)

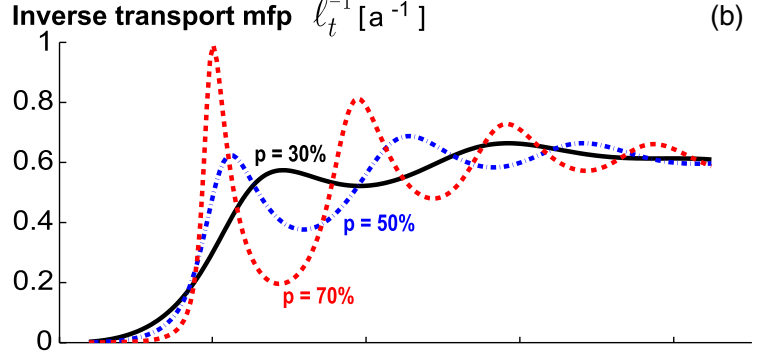

Scattering anisotropy factor $g$

(c)

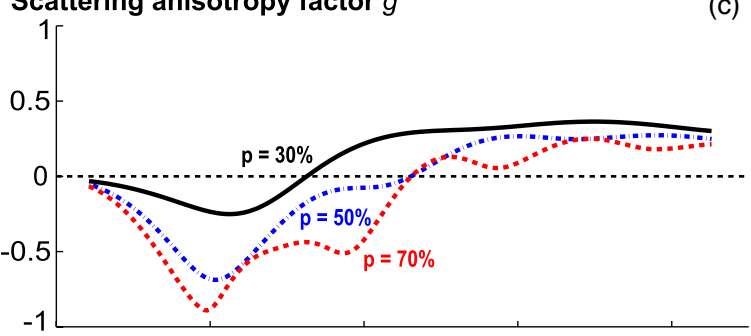

Localization length $\xi[$ a ]

(d)

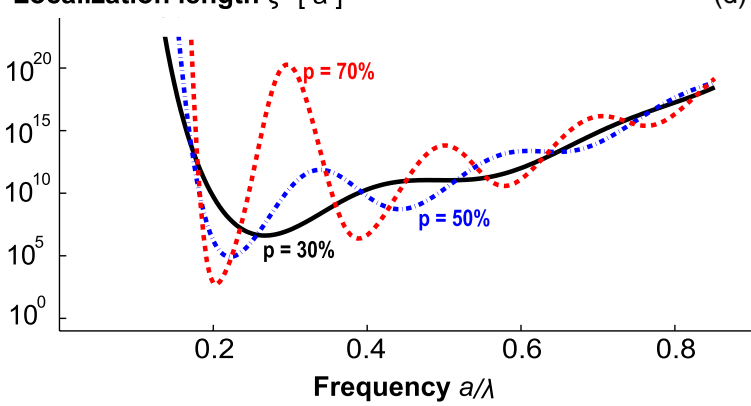

FIG. 2 (color online). Modified transport due to correlations. (a) Spectral and angular map of the "effective" differential scattering cross section $d \sigma^{\star} / d \theta$ for weakly $(p=30 \%)$ and strongly ( $p=70 \%$ ) correlated media. (b) Inverse transport mean free path $\ell_{t}^{-1}$, (c) scattering anisotropy factor $g$, and (d) localization length $\xi$ for different degrees of correlation $p$.

We therefore expect that a modification of the degree of correlation $p$ could lead to modifications of the localization length $\xi$ over orders of magnitude, making it possible to go from a quasiextended to a localized regime easily in finitesize systems. In Fig. 2(d), the localization length $\xi$ predicted semianalytically is shown in semi-log scale. The strong modulation of $\xi$ as a function of frequencies is striking. In the 2D correlated system, one goes from a regime in which $\xi$ is much larger than any realistic system (low frequencies) to a regime in which the two can be comparable (at $a / \lambda \approx 0.2$ ). Although we do not expect Eq. (5) to be quantitatively accurate [36], this huge photonic dispersion (variation of orders of magnitude within $\Delta \omega / \omega_{0}=0.2$ ), suggests that we could truly observe a dramatic variation of $\xi$ in real systems.

To test these predictions, we investigated the transport properties of the 2D correlated systems through numerical 2D finite-difference time-domain (FDTD) simulations, using a freely available software package [37]. We considered finite systems with the same structural parameters and open boundaries [squares of side $L=36 a$ surrounded by perfectly matched layers, see Fig. 3(a)]. The system was excited from a set of 225 randomly distributed dipole sources having an impulse with bandwidth of $0.02 \lambda / a$. Since the system is open, the energy density is expected to decay exponentially at long times as $U(t) \propto \exp [-\gamma t]$, where $\gamma$ is the decay constant. A change in the degree of correlation $p$ should yield a change in $\ell_{t}$ and thus in the average time needed for light to escape from the system. This is illustrated in Fig. 3(b), where, at a frequency $a / \lambda=0.21$, increasing $p$ yields a diminution of $\gamma$. The multiexponential decay at shorter times is due to the excitation of several modes in the structure which couple to the environment with different efficiency. The decay constants $\gamma$ were therefore obtained from exponential fits at sufficiently long times for various frequencies and degrees of correlation $p$, and an average was performed over six disorder realizations. Note that a proper statistical analysis of $\gamma$ would require more disorder realizations, which would be extremely time consuming. We have observed, however, that six disorder realizations are sufficient to show the increase of the decay constant as the degree of correlation increases, as reported below [Fig. 3(d)].

In Fig. 3(c), we show the average decay constants of the photonic structures with $p=30 \%, 50 \%$ and $70 \%$ as a function of the pulse excitation frequency, estimated from the numerical FDTD simulations. The effect of correlations on light transport is particularly clear. At frequencies close to $a / \lambda \approx 0.2, \gamma$ is strongly diminished due to a reduction of $\ell_{t}$ and at lower frequencies $(a / \lambda<0.17)$ one observes an increase of $\gamma$, in accordance with the increase of $\ell_{t}$ [see Fig. 2(b)]. Note that $\gamma$ drops over 2 orders of magnitude within a relative bandwidth of $\Delta \omega / \omega_{o}=0.2$.

It is also interesting to compare the values obtained numerically with those expected from the semianalytical approach within the diffusion approximation. The decay constant for diffuse light in a 2D system open along two directions is given by

$$
\gamma=\frac{2 \pi^{2} D}{\left(L+2 z_{e}\right)^{2}}
$$

where $z_{e}=(\pi / 4) \ell_{t}$ is the so-called extrapolated length $[38,39]$ (internal reflections are neglected), and $D=v_{e} \ell_{t} / 2$ is the diffusion constant with $v_{e}=c / n_{e}$ the energy velocity. According to Eq. (3), the optical thickness $L / \ell_{t}$ of our 
(a)
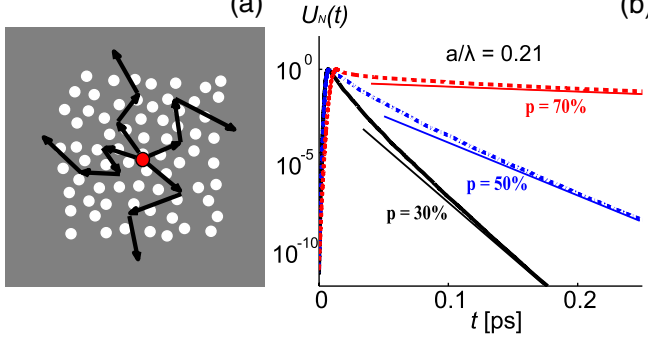

Decay rate $Y$ [c/a]

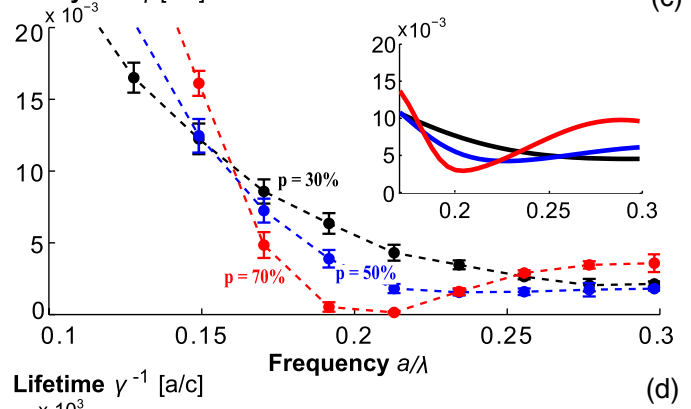

(d)

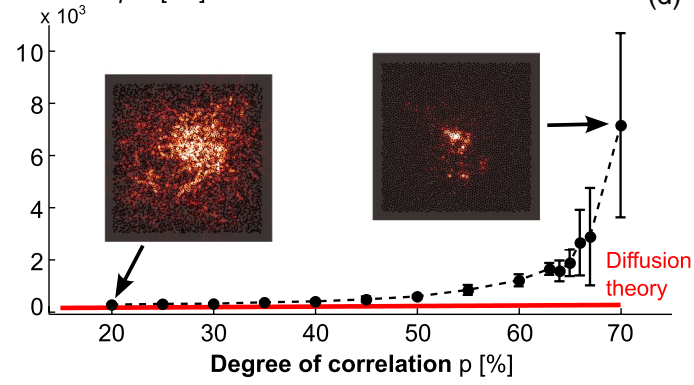

FIG. 3 (color online). Numerical FDTD simulations. (a) Sketch of the simulated system for the evaluation of the average decay constant of the modes. (b) Normalized integrated energy density $U_{n}(t)$ versus time at frequency $a / \lambda=0.21$, for different $p$. The straight lines are decaying exponentials, emphasizing the multiexponential decay observed at short times. The decay constant values $\gamma$ were estimated at long times. (c) Numerically estimated decay constants versus frequency as a function of $p$. The inset shows the decay constants evaluated semianalytically from Eq. (6), only for $L / \ell_{t} \geq 6$. (d) Numerically estimated average lifetime $\gamma^{-1}$ as a function of $p$ at frequency $a / \lambda=0.21$. The red line represents the prediction according to diffusion theory and the intensity maps are calculated for different $p$.

systems can be extremely small at very low frequencies, so that even less than a single scattering event can occur. In such a regime, Eq. (6) is not accurate [40]. Hence, we limit our analysis to frequency domains [inset in Fig. 3(c)] for which $L / \ell_{t} \geq 6$, so that the accuracy of diffusion theory is still reasonably good. While the trends of the decay constant as a function of frequency and degree of correlation are in good agreement, only a fair agreement is found quantitatively. The deviation for $p=70 \%$ in particular is marked (more than 1 order of magnitude). This discrepancy can be explained by considering that (i) the expression used to evaluate the transport mean free path [Eq. (3)] neglects completely recurrent scattering and near-field phenomena which are likely to occur in such dense systems (here, the filling fraction is $f=20 \%$ ), and (ii), more importantly for the strongly correlated system, the diffusion approximation disregards completely light localization phenomena, due to interference between multiply scattered waves, which are expected to yield a reduction of the diffusion constant.

To better appreciate the occurrence of light localization in these systems, we retrieve the average lifetime $\gamma^{-1}$ from the numerical data at frequency $a / \lambda=0.21$ for different $p$, as shown in Fig. 3(d). The clear, weakly fluctuating trend with varying $p$ indicates that six disorder realizations already provide reasonably converged results. The red line represents the prediction of diffusion theory according to Eqs. (3) and (6). A clear deviation between numerical calculations and diffusion theory occurs as $p$ increases. In particular, a dramatic increase of $\gamma^{-1}$ over an order of magnitude is observed for $p=70 \%$. Such a pronounced effect cannot be attributed solely to a reduction of $\ell_{t}$, since it would be captured by Eq. (6), but rather to Anderson localization of light. Localized modes are, on average, characterized by an exponentially decaying intensity distribution [41]. This implies, due to weak coupling between these modes and the environment, lifetimes on average that are much longer than those of quasiextended modes. Here, the reduction of $\ell_{t}$ with increasing $p$ yields a considerable variation of the localization length $\xi$, which eventually becomes shorter than the sample size $L$. This is, indeed, supported by the intensity maps shown in the insets of Fig. 3(d), calculated for a single steady-state source placed in the center of the system. Our interpretation is also corroborated by the observation of large variations of lifetimes for different realizations of disorder, as shown by the pronounced error bars in Fig. 3(d) for high $p$, which is expected in the localized regime. A transition from a quasi-extended regime $(\xi>L)$ to a localized regime $(\xi \leq L)$ has therefore been achieved by merely adding short-range correlations in the disordered system, keeping $f$ and $\varnothing$ unchanged. From these considerations, we can estimate that $\xi \leq L$, which is 1 order of magnitude smaller with respect to the prediction of Eq. (5).

To conclude, we have investigated how short-range correlations lead to considerable modifications of light transport and localization phenomena in 2D disordered photonic structures. Using a semianalytical approach for the structure factor of the correlated systems (due to Baus and Colot [32]) and a modified independent scattering approximation $[15,16]$, we have investigated how key transport quantities are affected by short-range structural correlations. We have found in particular that short-range correlations make it possible to increase and/or decrease the localization length by several orders of magnitude. This shows that it is possible to design structures that are very weakly scattering and strongly localizing at nearby frequencies. Twodimensional disordered systems in which the light transport and localization are finely controlled may find interest in the fundamental study of localization phenomena [5], the conception of planar random lasers [42], thin-film 
photovoltaic and lighting technologies [10], on-chip random spectrometers [14], or even help to reach the strong coupling regime with quantum dots or molecules $[43,44]$.

This work is supported by the Italian National Research Council (CNR) through the "EFOR" project, and the LABEX WIFI (Laboratory of Excellence within the French Program "Investments for the Future") under references ANR-10LABX-24 and ANR-10-IDEX-0001-02 PSL*. The research leading to these results has received funding from the European Research Council under the European Union's Seventh Framework Programme (FP7/2007-2013) / ERC grant agreement No. [291349].

*burresi@lens.unifi.it

${ }^{\dagger}$ Present address: Laboratoire Photonique, Numérique et Nanosciences (LP2N), UMR 5298, CNRS - IOGS -Université Bordeaux, Institut d'Optique d'Aquitaine, 33400 Talence, France.

[1] A. Ishimaru, Wave Propagation and Scattering in Random Media (Wiley-IEEE Press, New York, 1999).

[2] E. Akkermans and G. Montambaux, Mesoscopic Physics of Electrons and Photons (Cambridge University Press, Cambridge, England, 2007), 1st ed.

[3] P. Sheng, Introduction to Wave Scattering, Localization and Mesoscopic Phenomena (Springer, New York, 2010), 2nd ed.

[4] D. S. Wiersma, Nat. Photonics 7, 188 (2013).

[5] F. Riboli, P. Barthelemy, S. Vignolini, F. Intonti, A. De Rossi, S. Combrie, and D. S. Wiersma, Opt. Lett. 36127 (2011).

[6] P. D. García, S. Stobbe, I. Söllner, and P. Lodahl, Phys. Rev. Lett. 109, 253902 (2012).

[7] M. Burresi, V. Radhalakshmi, R. Savo, J. Bertolotti, K. Vynck, and D. S. Wiersma, Phys. Rev. Lett. 108, 110604 (2012).

[8] T. Strudley, T. Zehender, C. Blejean, E. P. A. M. Bakkers, and O. L. Muskens, Nat. Photonics 7, 413 (2013).

[9] L. Sapienza, H. Thyrrestrup, S. Stobbe, P. D. Garcia, S. Smolka, and P. Lodahl, Science 327, 1352 (2010).

[10] K. Vynck, M. Burresi, F. Riboli, and D. S. Wiersma, Nat. Mater. 11, 1017 (2012).

[11] M. Burresi, F. Pratesi, K. Vynck, M. Prasciolu, M. Tormen, and D. S. Wiersma, Opt. Express 21, A268 (2013).

[12] A. Oskooi, P. A. Favuzzi, Y. Tanaka, H. Shigeta, Y. Kawakami, and S. Noda, Appl. Phys. Lett. 100, 181110 (2012).

[13] C. Lin, L. J. Martínez, and M. L. Povinelli, Opt. Express 21, A872 (2013).

[14] B. Redding, S. F. Liew, R. Sarma, and H. Cao, Nat. Photonics 7, 746 (2013).

[15] S. Fraden and G. Maret, Phys. Rev. Lett. 65, 512 (1990).

[16] P. M. Saulnier, M. P. Zinkin, and G. H. Watson, Phys. Rev. B 42, 2621 (1990).

[17] L. F. Rojas-Ochoa, J. Mendez-Alcaraz, J. Sáenz, P. Schurtenberger, and F. Scheffold, Phys. Rev. Lett. 93, 073903 (2004).

[18] M. Reufer, L. F. Rojas-Ochoa, S. Eiden, J. J. Sáenz, and F. Scheffold, Appl. Phys. Lett. 91, 171904 (2007).

[19] J. Yang, C. Schreck, H. Noh, S.-F. Liew, M. I. Guy, C. S. O'Hern, and H. Cao, Phys. Rev. A 82, 053838 (2010).
[20] H. Yin, B. Dong, X. Liu, T. Zhan, L. Shi, J. Zi, and E. Yablonovitch, Proc. Natl. Acad. Sci. U.S.A. 109, 10798 (2012).

[21] K. Edagawa, S. Kanoko, and M. Notomi, Phys. Rev. Lett. 100, 013901 (2008).

[22] M. Florescu, S. Torquato, and P. J. Steinhardt, Proc. Natl. Acad. Sci. U.S.A. 106, 20658 (2009).

[23] S. Imagawa, K. Edagawa, K. Morita, T. Niino, Y. Kagawa, and M. Notomi, Phys. Rev. B 82, 115116 (2010).

[24] M. Rechtsman, A. Szameit, F. Dreisow, M. Heinrich, R. Keil, S. Nolte, and M. Segev, Phys. Rev. Lett. 106, 193904 (2011).

[25] T. Sperling, W. Bührer, C. M. Aegerter, and G. Maret, Nat. Photonics 7, 48 (2013).

[26] F. Pratesi, M. Burresi, F. Riboli, K. Vynck, and D. S. Wiersma, Opt. Express 21, A460 (2013).

[27] M. Skoge, A. Donev, F. H. Stillinger, and S. Torquato, Phys. Rev. E 74, 041127 (2006).

[28] B. D. Lubachevsky and F. H. Stillinger, J. Stat. Phys. 60, 561 (1990).

[29] J. C. M. Garnett, Phil. Trans. R. Soc. A 205, 237 (1906).

[30] A.H. Sihvola, Electromagnetic Mixing Formulas and Applications (IET, London, 1999), ISBN 9780852967720.

[31] S. Torquato, Random Heterogeneous Materials (Springer, New York, 2001), corrected edition.

[32] M. Baus and J. L. Colot, Phys. Rev. A 36, 3912 (1987).

[33] H. C. van de Hulst, Light Scattering by Small Particles (Dover Publications, New York, 1981).

[34] Note that the expression used in Refs. [19,45] applies exclusively to $3 \mathrm{D}$ systems.

[35] R. Gómez-Medina, L. S. Froufe-Pérez, M. Yépez, F. Scheffold, M. Nieto-Vesperinas, and J. J. Sáenz, Phys. Rev. A 85, 035802 (2012).

[36] Equation (5) is derived from a renormalization of the diffusion constant in which it is assumed that the correction term is small [3]. Also, $\ell_{t}$ is obtained neglecting recurrent scattering and near-field interactions. Since minute variations of $\ell_{t}$ lead to large variations in $\xi$, we do not expect to have a quantitatively accurate estimation of $\xi$ for our dense systems.

[37] A. F. Oskooi, D. Roundy, M. Ibanescu, P. Bermel, J. D. Joannopoulos, and S. G. Johnson, Comput. Phys. Commun. 181, 687 (2010).

[38] M. C. W. van Rossum and T. M. Nieuwenhuizen, Rev. Mod. Phys. 71, 313 (1999).

[39] B. Payne, A. Yamilov, and S. E. Skipetrov, Phys. Rev. B 82, 024205 (2010).

[40] R. Elaloufi, R. Carminati, and J. Greffet, J. Opt. Soc. Am. A 21, 1430 (2004).

[41] The intensity distribution of a localized mode in a single disorder realization can often deviate strongly from a profile with a clear exponential decay.

[42] J.-K. Yang, H. Noh, S. F. Liew, M. J. Rooks, G. S. Solomon, and H. Cao, Phys. Rev. A 84, 033820 (2011).

[43] H. Thyrrestrup, S. Smolka, L. Sapienza, and P. Lodahl, Phys. Rev. Lett. 108, 113901 (2012).

[44] A. Cazé, R. Pierrat, and R. Carminati, Phys. Rev. Lett. 111, 053901 (2013).

[45] H. Noh, J.-K. Yang, S. F. Liew, M. J. Rooks, G. S. Solomon, and H. Cao, Phys. Rev. Lett. 106, 183901 (2011). 